\title{
Study on the Role of Sit-ups on the Nonspecific Low Back Pain of Postpartum Women
}

\author{
Liang $\mathrm{LI}^{1, \text { a }}$, Li-heng ZHANG ${ }^{1, \mathrm{~b}}$, Yue ZHOU ${ }^{2, \mathrm{c}}$ \\ ${ }^{1}$ Jilin province people's hospital, Changchun, 130021 \\ ${ }^{2}$ Xinqiao Hospital, Third Military Medical University, Chongqing, 400037 \\ atadwll@126.com, bhappy_zlh@126.com, chappyzhou@vip.163.com.
}

Keywords: Non-specific low back pain, sit-ups, Postpartum women

\begin{abstract}
Our objective is to verify the Role of sit-ups exercises on the non-specific low back pain of the postpartum women. 234 patients were randomly divided into two groups: group A and group B, with the same 117 patients in each group. Group A received sit-ups training and group B didn't. All 234 patients were followed up. The follow-up period was 12-17 months and the average follow-up time was 15months. According to VAS scores, counting the low back pain incidence and prevalence at 3 and 12 months postpartum. Through T-test by SPSS software, the prevalence between the two groups has significant difference $(t=12.098, p<0.05)$ in 3 and 12 months postpartum. It is found that sit-ups exercises can effectively reduce the postpartum non-specific low back pain prevalence. The pain prevalence of group A experienced spontaneous delivery was significantly lower than that of group B underwent uterine-incision delivery, which suggests that the abdominal muscles may play an important role for non-specific low back pain.
\end{abstract}

\section{Introduction}

Low back pain is one of the diseases with the largest amount in orthopedic outpatient treatment. There are three common pathogenic factors: Discogenic low back pain, Myogenic low back pain and low back pain due to lumbar facet joint ${ }^{[1,2]}$ and the non-specific low back pain. Non-specific low back pain accounts for $40 \%-55 \%{ }^{[3-5]}$. In postpartum women, it may exist in a higher prevalence of low back pain.

Clinical symptoms occurred mainly in the lower back, lumbar, sacroiliac, buttocks or legs with a subjective feeling of low back pain (Low Back Pain, LBP)[6]. Postpartum women are most frequently involved by nonspecific low back pain [7-9].Postpartum women have weaker abdominal muscles and their abdominal pressure is lower. This severely affects the stability of the spine. Sit-ups training can strengthen abdominal muscles, so as to strengthen abdominal pressure and increase spinal stability[10-12]. Therefore, sit-ups may be an effective way to prevent non-specific low back pain of postpartum women.234 postpartum women were selected for studying from January 2011 to June 2013. Good effects were achieved and report was gotten as follows.

\section{Training Procedure}

General Material: 234 patients (225 primipara cases and 9 multipara cases) aged from 23 to 32 years old with the e mean age 27.5 years. All patients came from obstetrics. 123 cases experienced spontaneous delivery, 111 cases underwent uterine-incision delivery. All patients were in postpartum 1-2 weeks to begin to practice, without suffering from any low back pain. Inclusion criteria: (1) Without obvious low back pain symptoms. (2) Without organic lumbar diseases, including lumbar disc herniation, lumbar spinal stenosis, congenital spinal disease and so on; (3) VAS score is zero. The patients were randomly divided into two groups: A and B group, and the number of each group is the same 117. A group is a training group (A1 group is 58 cases of spontaneous delibery, A2 group is 59 cases of uterine-incision delivery.), and B group is non-training group (B1 group is 65 cases of spontaneous delibery, B2 group is 52 cases of 
uterine-incision delivery.). There is no significant difference between the any two-group in the caesarean section or not and the age.

Sit-ups' right approach: According to Stamford (1997)[14], sit-ups' right approach is as follows: the body lying on the mat, knees bent at 90 degrees, feet flat on the ground. Do not fix the foot on the ground (for example, handle the ankle by a companion), otherwise thigh and hip flexor is added to work, thus reducing the workload of the abdominal muscles. Furthermore, the straight leg sit-ups will increase the burden on the back, easily brings about the back damage. Beginners can handle against the sides of the body. Do not put your hands placed on the head of his fingers crossed behind to avoid exertion strained of neck muscles, and it also reduces the workload of the abdominal muscles. When the abdominal muscles pulled up the body, you should exhale. This ensures that the deeper abdominal muscles are also involved. The body rises from 10 to $20 \mathrm{~cm}$ from the ground. It should tighten the abdominal muscles and pause, and slowly drop the body back into place. When the back lies on the ground, the next cycle of action can begin. Beginners should avoid doing too much frequency of sit-ups. You can try to do five times when initially performed, and then each practice adds up one time, until it reaches 15 times or so, then you can try to do the three groups so far. (Fig. 1).

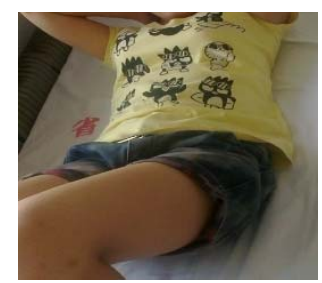

Fig. 1. Sit-ups’ right approach

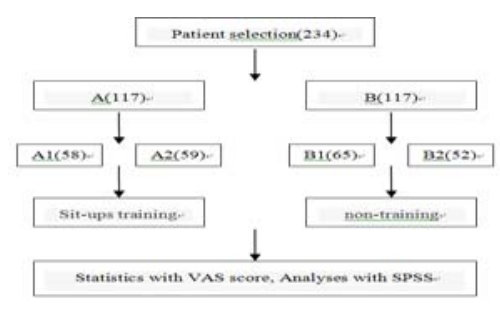

Fig. 2. Flow Chart

Low back pain classification: Pain in the low back was measured using the VAS scale (Table 1): Mild pain: 0-3 VAS score; Moderate pain: 4-6 VAS score; Severe pain: 7-10 VAS score. Score (0-10): 0 points, painless; 3 points or less, there is a slight pain, the patient can tolerate, do not affect the rest; 4 to 6 points, the patients feel pain and sleep affected, and can still endure, should be given a certain processing; 7 to 10 points, unbearable pain in patients, affecting appetite, affect sleep. Clinically, the patient should not hurt to such extent .

Table 1. VAS scale

\begin{tabular}{|l|l|l|}
\hline Mild pain & Moderate pain & Severe pain \\
\hline $0-3$ & $4-6$ & $7-10$
\end{tabular}

Methods: A group: According to the patient postpartum recovery, all patients began to practice after 1-2 weeks of production. First week of action aimed to being able to make a standard sit-ups action. Because of postpartum abdominal relaxation, mothers are often not able to take the initiative to sit up. They can get the proper help from others. To straighten knees is also a good way. Second week of action aimed to being able to make 5 standard sit-ups action. Third week of action aimed to being able to make 15 standard sit-ups action each day. From the fourth week they began to make three times 15-standard-sit-ups-action each day. Observe the situation of the low back pain on the time of 1,3 and 12 months postpartum.

B group: Without training, observe the situation of the low back pain on the time of 1,3 and 12 months postpartum.The VAS scores between A and B, A1 and A2 or B1 and B2 were statistically analyzed by SPSS12.0 software. 


\section{Results}

All 234 patients were followed up. The follow-up time was 12-17 months, and the average follow-up time was 15months. According to VAS scores, count the low back pain incidence and prevalence in the time of , 3 and 12 months postpartum ( Table 2. ) .

Table 2. Disease incidence in the time of 1,3,12 months postpartum. according VAS scores.

\begin{tabular}{|l|l|l|l|l|l|l|l|l|l|}
\hline & \multicolumn{3}{|l}{$\begin{array}{l}\text { Posttraining score } \\
(1 \text { month later })\end{array}$} & \multicolumn{2}{l|}{$\begin{array}{l}\text { Posttraining score } \\
\text { (3 month later) }\end{array}$} & \multicolumn{2}{l|}{$\begin{array}{l}\text { Posttraining score } \\
(12 \text { month later })\end{array}$} \\
\hline VAS & $0-3$ & $4-6$ & $7-10$ & $0-3$ & $4-6$ & $7-10$ & $0-3$ & $4-6$ & $7-10$ \\
\hline A & 11 & 5 & 1 & 7 & 2 & 0 & 2 & 2 & 0 \\
\hline A1 & 3 & 1 & 0 & 1 & 1 & 0 & 1 & 1 & 0 \\
\hline A2 & 8 & 4 & 1 & 6 & 1 & 0 & 1 & 1 & 0 \\
\hline B & 7 & 9 & 1 & 10 & 21 & 1 & 18 & 28 & 6 \\
\hline B1 & 2 & 3 & 0 & 3 & 8 & 0 & 7 & 10 & 2 \\
\hline B2 & 5 & 6 & 1 & 7 & 13 & 1 & 11 & 18 & 4 \\
\hline
\end{tabular}

Table 3. Disease incidence and prevalence in the time of 1, 3, 12 months postpartum between A and B

\begin{tabular}{|l|l|l|l|}
\hline & 1 month later & 3 month later & 12 month later \\
\hline A & $17(13.7 \%)$ & $9(7.7 \%)$ & $4(3.4 \%)$ \\
\hline B & $17(13.7 \%)$ & $32(27.4 \%)$ & $52(44.4 \%)$ \\
\hline
\end{tabular}

Table 3 shows the prevalence decreased in A groups, but it increased in B groups. Through T-test by SPSS software, the prevalence in the time of 1 month postpartum between A and B groups has no significant difference $(\mathrm{t}=13.052, \mathrm{p}>0.05)$. But the prevalence between the two groups has significant difference $(t=12.098, p<0.05)$ in the time of 3,12 months.

Table 4. Disease incidence in 1,3,12 months postpartum between A1 and A2

\begin{tabular}{|l|l|l|l|}
\hline & 1 month later & 3 month later & 12 month later \\
\hline A1 & $4(7.0 \%)$ & $2(3.4 \%)$ & $2(3.4 \%)$ \\
\hline A2 & $13(22.0 \%)$ & $7(11.9 \%)$ & $2(3.4 \%)$ \\
\hline
\end{tabular}

Table 4 shows the prevalence decreased in A1 and A2 groups. Through T-test by SPSS software, the prevalence in the time of 1, 3, 12 months month postpartum between A1 and A2 groups has significant difference $(\mathrm{t}=11.472, \mathrm{p}<0.05)$.

The prevalence increased in B1 and B2 groups. Through T-test by SPSS software, the prevalence in the time of $1,3,12$ months month postpartum between B1 and B2 groups has significant difference $(\mathrm{t}=13.011, \mathrm{p}<0.05)$.

\section{Conclusion}

Sit-ups exercises can effectively reduce postpartum non-specific low back pain prevalence. It is found that the pain prevalence of women experienced spontaneous delivery was significantly lower than that of women underwent uterine-incision delivery. This suggests that abdominal muscles may play an important role for non-specific low back pain.

\section{References}

[1] Li Liang, Zhou Y.,et al. Comparision of Two Blocking Solutions in The Treatment of the Lumer Facet Joint of OSteorathritis, Journal of Investigative Medicine, 61(4)S(2013), p.S11-S12.

[2] Liang LI, Li-heng ZHANG, Yue ZHOU. C-arm X-ray Machine Guided Blocking Treatment of Lumbar Facet Joint Osteoarthritis[J]. Advanced Materials Research (2013), p.4549-4552.

[3] ZHANG Li-heng, LI Liang, LI Xiao-feng, et al. A experimental study on chemosensitivity to Osteosarcoma in vitro. Chinese Journal of Laboratory Diagnosis. 17(1)(2013), p.100-102. 
[4] Li-heng ZHANG, Liang LI. Detection of Differentially Expressed Proteins of Capsulation-resistant and Sensitive Osteosarcoma Tissue. Advanced Materials Research. (2013), p.4553-4556.

[5] ZHENG Wei-dong, LI Liang, ZHAO Yun-he. Clinical study on the vertebral plasty for the treatment of osteoporotic vertebral co3mpressed fractures in elderly. China J Orthop \& Trauma, 21(6)(2008), p.405-406.

[6] Anderson GB. Epidemiologica1 features of chronic low back pain. Lancet, $354 \quad$ (1999), p.581-585.

[7] Ashton-Miller JA, Schultz AB. Spine instability and segmental hypermobility biomechanics: A call forthe definition and standard use of terms. Seminars in Spine Surgery 3(1991), p.136-148.

[8] Baratta R, Solomonow M, Zhou BH, Letson D, Chuinard R, D'Ambrosia R. Muscular coactivation: The role of the antagonist musculature in maintaining knee stability. Am J Sports Med 6(1988), p. 113-122.

[9] Bean JC, Chaffin DB, Schultz AB. Biomechanical model calculation of muscle contraction forces: A double linear programming method. J Biomech 21(1988), p.59-66.

[10] Bergmark A. Stability of the lumbar spine: A study in mechanical engineering. Acta Orthop Scand 230(Suppl) (1989), p.S1-54.

[11] Bogduk N, Pearcy M, Hadfield G. Anatomy and biomechanics of psoas major. Clin Biomech 7(1992), p.109-119.

[12] Cholewicki J, McGill SM. Mechanical stability of the in vivo lumbar spine: Implications for injury and chronic low back pain. Clin Biomech 11 (1996), p.1-15.

[13] Stamford, B. A. The right way to do sit-ups. Physician and Sportsmedicine, 1997, 25(6): 111-112. 